\title{
Modelagem de Dados Geofísico-espaciais por Lógica Fuzzy Aplicada ao Distrito Ferrífero Nova Aurora, Norte de Minas Gerais
}

\author{
Geophysical-spatial Data Modeling using Fuzzy Logic Applied to Nova Aurora Iron District, \\ Northern Minas Gerais State, Southeastern Brazil
}

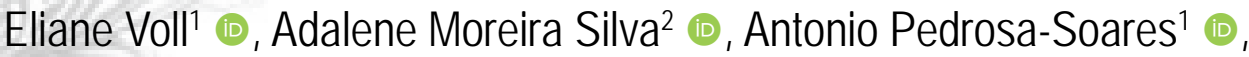 Ranufo Arimatéia ${ }^{3}$ \& Francisco Teixeira Vilela ${ }^{1}$}

\author{
${ }^{1}$ Universidade Federal de Minas Gerais, Programa de Pós-Graduação em Geologia, CPMTC-IGC, Belo Horizonte, MG, Brasil \\ ¿Universidade de Brasília, Brasília, Instituto de Geociências, DF, Brasil \\ ${ }^{3}$ Sul Americana de Metais S/A, Belo Horizonte, MG, Brasil \\ E-mails: eliane.voll@gmail.com; adalene@unb.br; pedrosasoares@gmail.com; ranufo.arimateia@sammetais.com.br; \\ francisco.vilela@cprm.gov.br
}

\section{Resumo}

Integração e modelagem de dados geofísicos, geológicos e de sensores remotos, em sistemas de informação geográfica (SIG), têm sido usadas com sucesso em mapeamento geológico e exploração mineral, em todo o mundo, particularmente em áreas com escassos afloramentos rochosos e extensas coberturas cenozoicas. Situado no norte de Minas Gerais, o Distrito Ferrífero Nova Aurora engloba metadiamictitos do Grupo Macaúbas ricos em ferro, relacionados a uma glaciação global do Criogeniano. O distrito, cujas reservas totais superam vinte bilhões de toneladas de minério de ferro $(15-60 \% \mathrm{Fe})$, situa-se numa área de extensas chapadas, largamente cobertas por espessos regolitos e sedimentos cenozoicos, escondendo os depósitos de ferro. Os metadiamictitos ricos em ferro contêm quantidades variáveis de hematita (predominante) e magnetita (concentrada em zonas de cisalhamento) na matriz foliada que também inclui quartzo, muscovita, feldspato, granada, clorita e carbonato, dentre outros minerais. Mapas regionais de favorabilidade, baseados em Lógica Fuzzy, apresentados em artigo anterior publicado pelos autores, indicaram grande número de alvos, alguns deles em prospecção avançada pela Companhia Sul Americana de Metais (SAM). O presente artigo focaliza um desses alvos trabalhados pela SAM, visando testar a aplicação daquela metodologia de integração e modelagem de dados geofísicos-espaciais sobre uma área reduzida. O modelo resultante foi validado: i) pelo mapa geológico de detalhe, elaborado durante o trabalho de prospecção; ii) pela distribuição de afloramentos de minério que coincide com as áreas de maior favorabilidade no modelo e; iii) pelas profundidades calculadas, por deconvolução de Euler, para as fontes magnéticas que são compatíveis com as espessuras do corpo de minério nas seções geológicas controladas por furos de sonda. Os dados e método utilizados foram eficazes na redução da ambiguidade da informação e o modelo integrado restringe as áreas mais favoráveis à ocorrência do minério de ferro.

Palavras-chave: Minério de ferro; Diamictito; Grupo Macaúbas 


\begin{abstract}
Integration and modeling of geophysical, geological, and remote sensor data, using geographic information system (GIS), have been successfully applied in geological mapping and mineral prospecting worldwide, particularly in areas with scarce outcrops and extensive Cenozoic covers. In northern Minas Gerais State (SE Brazil), the Nova Aurora Iron District encompasses iron-rich metadiamictites of the Macaúbas Group, related to a global Cryogenian glaciation. The district, with total reserves exceeding twenty billion tons of iron ore $(15-60 \mathrm{wt} \% \mathrm{Fe})$, is located in an area of extensive plateaus (chapadas) largely covered by thick regoliths and Cenozoic sediments that hide the iron deposits. The iron-rich metadiamictites contain variable amounts of hematite (largely prevalent) and magnetite (concentrated in shear zones) in a foliated matrix which also includes quartz, mica, feldspar, garnet, chlorite, and carbonate, among other minerals. Regional favorability maps, based on Fuzzy Logic modeling, published in a previous article by the authors, indicated a large number of targets, some of them in advanced prospecting by the Companhia Sul Americana de Metais (SAM). The present paper focuses on one of these SAM targets, aiming to test the application of data integration and Fuzzy Logic modeling on a small area. The resulting model was validated by the: i) geological detail map, developed during the prospecting work; ii) distribution of ore outcrops that coincides with the sites of greatest favorability in the model; and iii) calculated depths for the magnetic sources, by using Euler deconvolution, that are compatible with the thickness of the ore body in the geological sections controlled by data from drill holes. The data and method used were effective in reducing the information ambiguity so that the integrated model proved to be efficient in restricting the most favorable areas for iron ore occurrence.
\end{abstract}

Keywords: Iron ore; Diamictite; Macaúbas group

\section{Introdução}

Modelagem de dados geofísicos, geológicos e de sensoriamento remoto tem sido usada com sucesso para identificar alvos minerais e auxiliar no mapeamento geológico em todo o mundo, a exemplo de modelagem prospectiva voltada para depósitos de ferro, usando técnicas de análise espacial em Sistema de Informação Geográfica SIG (Andrade, Silva, \& Souza Filho 2014; Crósta, Bicho, \& Ferreira 1994; Voll, Silva \& Pedrosa-Soares 2020; Zacchi, Silva \& Rolim 2010).
Com o objetivo de rastrear depósitos de ferro do Grupo Macaúbas (Neoproterozoico) em alvos situados em planaltos (chapadas) cenozoicos no norte de Minas Gerais, aplicamos o método baseado em lógica fuzzy para integração de dados geológicos, aerogeofísicos e de sensoriamento remoto sobre uma área de $66,6 \mathrm{~km}^{2}$ do Distrito Ferrífero Nova Aurora (Figura 1). O Distrito Ferrífero Nova Aurora é caracterizado por formações ferríferas diamictíticas $(\mathrm{Fe}>15 \%)$ e metadiamictitos ferruginosos (Fe entre $5 \mathrm{e}$ $15 \%$ ), variavelmente ricos em hematita e/ou magnetita,

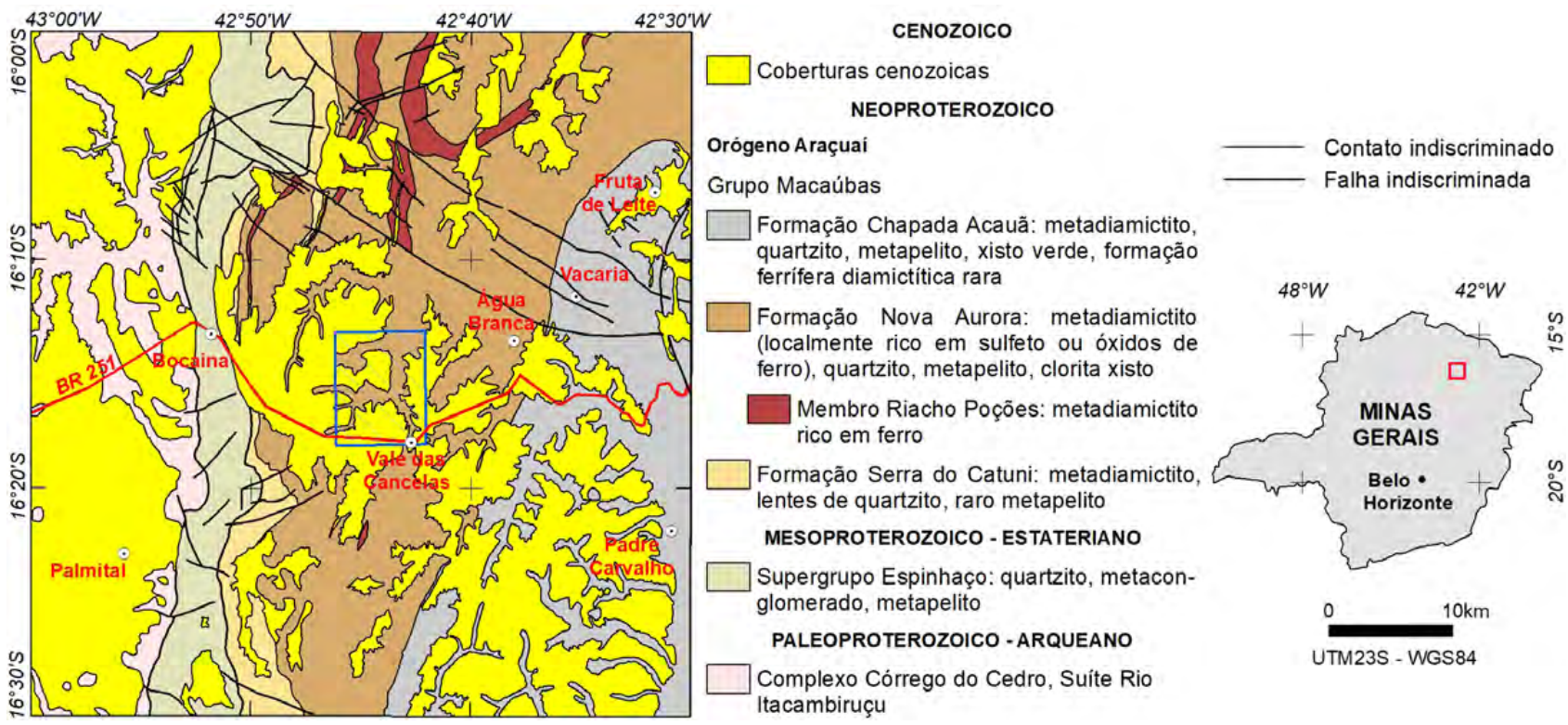

Figura 1 Mapa geológico simplificado da Folha Padre Carvalho (modificado de Mourão \& Grossi Sad 1997). Retângulo azul indica a área selecionada para este estudo. 
que compõem o Membro Riacho Poções da Formação Nova Aurora, Grupo Macaúbas (Pedrosa-Soares et al. 2011; Vilela et al. 2014, 2021; Viveiros et al. 1978).

O modelo prospectivo proposto neste trabalho é baseado em combinações de dados espaciais que reduzem a ambiguidade de interpretações que geralmente surgem quando a análise é realizada exclusivamente por métodos analógicos. A área potencial foi selecionada de acordo com conhecimento prévio e por um conjunto de informações de entrada disponíveis que permitem suas delimitações, atendendo a critérios predefinidos (An, Moon \& Rencz 1991; Bonham -Carter 1994; Raines, Sawatzky \& BonhamCarter 2010). No modelo de lógica fuzzy, aplicado neste trabalho, os mapas são classificados de acordo com seu grau de pertinência, com valores crescendo continuamente entre 0 e 1 , onde 1 é o valor máximo de favorabilidade em relação ao alvo estudado. Este modelo é adequado para determinar as classes de caracterização quando limites rígidos não podem ser definidos entre conjuntos de dados (An, Moon \& Rencz 1991; Bonham-Carter 1994).

A partir da modelagem, este artigo apresenta um modelo prospectivo para área selecionada (Figura 1), com base nas relações espaciais entre rochas ricas e pobres em ferro no Distrito Ferrífero Nova Aurora, conforme metodologia aplicada em escala regional por Voll, Silva \&
Pedrosa-Soares (2020). Neste estudo de caso, comparam-se os resultados encontrados pelo modelo com verificações em campo, e com o mapa geológico de detalhe (que inclui informação de 450 furos de sondagem rotativa), elaborado pela empresa Sul Americana de Metais S.A. (SAM), além das áreas de requerimento de lavra da SAM S.A. (Agência Nacional de Mineração 2019). Utilizando esse modelo, pretende-se, de forma suplementar, determinar se também pode ser considerado eficiente para estudos locais.

\section{Caracterização da Área}

A área de estudo abranje $66,6 \mathrm{~km}^{2}$ e se localiza no limite dos municípios de Padre Carvalho e Grão Mogol, MG (Figura 2).

Coberturas elúvio-coluvionares ocorrem em grande parte da área, ocultando largamente as rochas da Formação Nova Aurora, inclusive aquelas ricas em ferro, as quais afloram em vales internos e limitantes do planalto (Figura 1). A geomorfologia e geologia regionais estão descritas em Mourão \& Grossi Sad (1997); Pedrosa-Soares et al. (2011); Saadi (1995) e Voll, Silva \& Pedrosa-Soares (2020). As rochas que ocorrem em afloramentos e testemunhos de sondagem (Figura 3), na área de estudo estão sumariamente descritas adiante (Vilela 2010, Vilela et al. 2021).
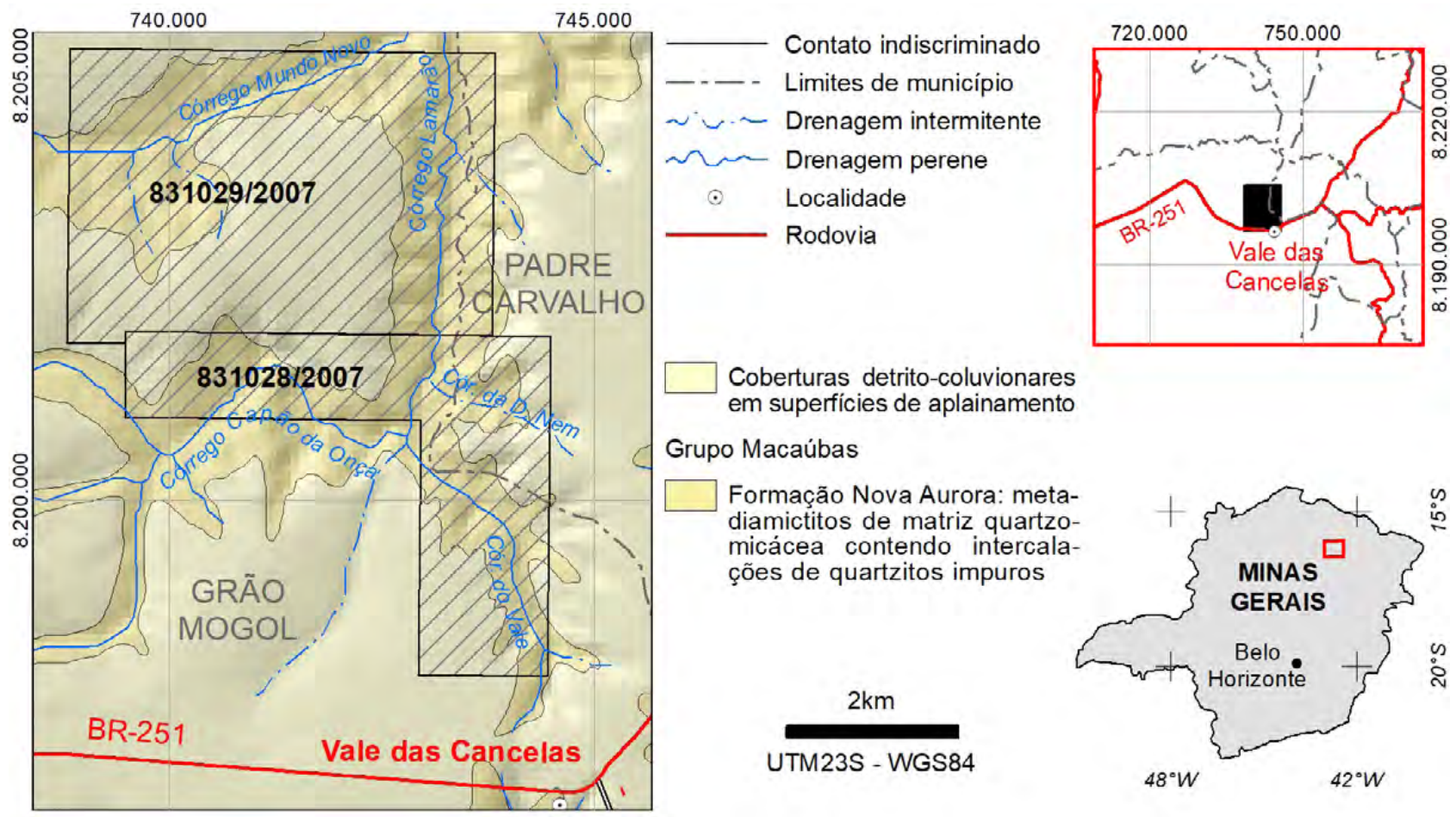

Coberturas detrito-coluvionares em superfícies de aplainamento

\section{Grupo Macaúbas}

Formação Nova Aurora: metadiamictitos de matriz quartzomicácea contendo intercalações de quartzitos impuros

Figura 2 Localização da área de estudo, mostrando blocos de prospecção da SAM (Agência Nacional de Mineração 2019) sobre a geologia simplificada da Folha Padre Carvalho (ver Figura 1). 


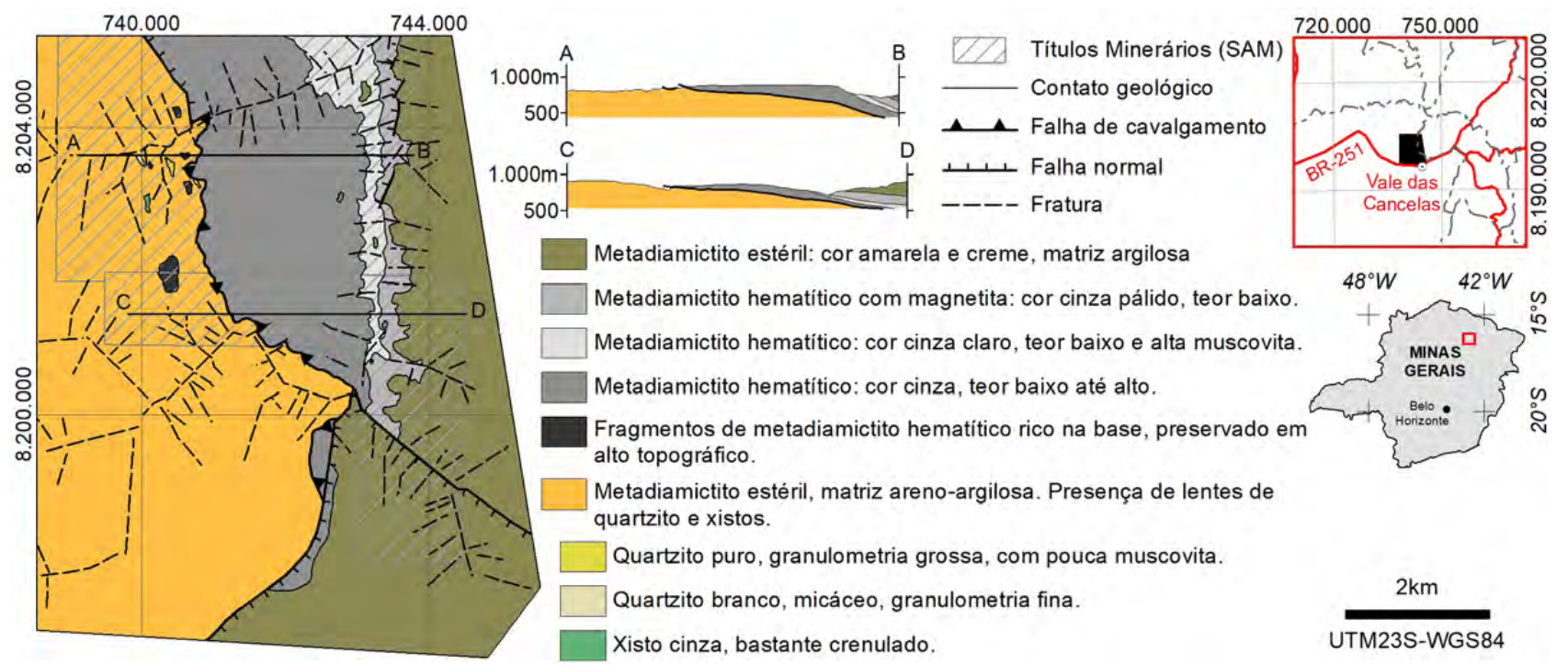

Figura 3 Mapa geológico da área de estudo (modificado de Sul Americana de Metais 2013).

\subsection{Metadiamictito Estéril}

É a rocha encaixante dos depósitos de ferro, composto de ampla variedade composicional de clastos, desde matacões a seixos, suportados por matriz foliada, variavelmente rica em quartzo e micas.

\subsection{Metadiamictito Hematítico e Formação Ferrífera Metadiamictitica Rica em Hematita}

A diferença entre estas duas rochas é apenas o teor em ferro total que, quando maior que $15 \%$ caracteriza a formação ferrífera, e entre 5 e $15 \%$, corresponde ao metadiamictito hematítico ou ferruginoso (Figura 4). A quantidade de hematita da matriz desses metadiamictitos varia entre $5 \%$ e $60 \%$. Hematita e micas ocorrem em concentrações inversamente proporcionais, observandose em campo a clara diminuição na concentração de hematita rumo ao topo mais enriquecido em mica. A hematita, incipientemente magnética, ocorre disseminada na matriz do metadiamictito, mas se concentra em lâminas, bandas e lentes correspondentes à foliação regional e clivagem de crenulação. Magnetita, parcial a totalmente martitizada, geralmente não passa de $2 \%$, exceto em zonas de cisalhamento onde ocorre enriquecimento em magnetita.
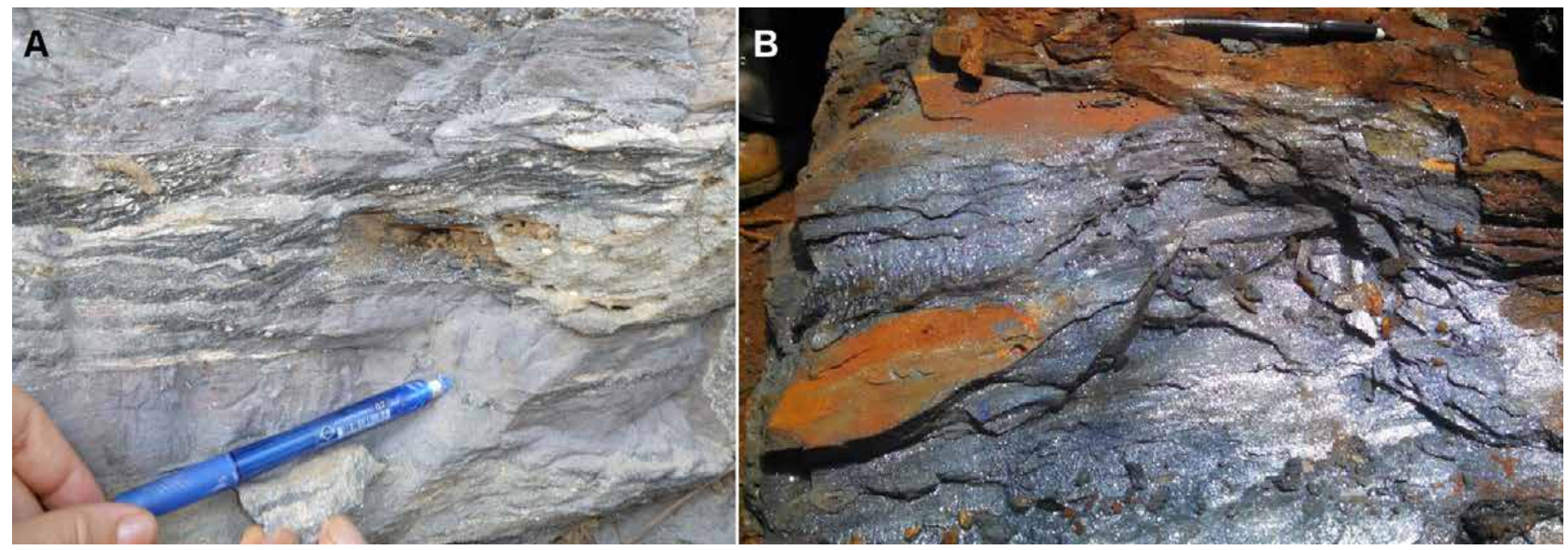

Figura 4 A. Formação ferrífera metadiamictítica rica em hematita (UTM23S-WGS84, 741.246E, 8.201.332N); B. Bloco de hematita especular à beira da estrada, contendo níveis quase inteiramente compostos por hematita e magnetita (UTM23S-WGS84, 740.468E, 8.201.919N). 


\subsection{Metadiamictito Magnetítico e Formação Ferrífera Metadiamictítica Rica em Magnetita}

Revelados por furos de sondagem, também são diferenciados apenas pelo teor em ferro total. Apresentam tonalidade cinza mais escura que os termos hematíticos e se caracterizam pelo aumento progressivo na quantidade de magnetita em relação à de hematita. Pode ser bandado, com bandas de cor cinza, ricas em magnetita, intercaladas com bandas mais ricas em quartzo e muscovita. A quantidade de hematita especular aumenta nas bandas ricas em magnetita. Quando apresenta tons esverdeados, o metadiamictito magnetítico mostra aumento na concentração de clorita e biotita.

\section{Dados, Métodos e Resultados}

Para a modelagem, os dados aerogeofísicos e de sensoriamento remoto foram trabalhados em ambiente SIG (Sistema de Informações Geográficas), após terem seus parâmetros cartográficos homogeneizados para a projeção Universal Transversa de Mercator (UTM), zona $23 \mathrm{~S}$ e datum horizontal WGS84.

\subsection{Dados Aerogeofísicos}

Os dados aerogeofísicos utilizados foram os da Área 8 (Minas Novas - Riacho dos Machados - Espinosa; Companhia de Desenvolvimento de Minas Gerais Secretaria de Desenvolvimento Econômico - Companhia de Pesquisa de Recursos Minerais 2006), que incluem levantamentos gamaespectrométricos e magnéticos em alta resolução ao longo de $87.212,45 \mathrm{~km}$, com intervalo entre linhas de vôo e linhas de controle de 0,5 e 10,0 km, nas direções N25W e N65E, respectivamente. Os intervalos entre as medições geofísicas consecutivas foram de $0,1 \mathrm{~s}$ (magnetômetro) e 1,0s (espectrômetro). A altura média de vôo foi de $100 \mathrm{~m}$, a uma velocidade média de $280 \mathrm{~km} / \mathrm{h}$.

\subsubsection{Gradiente Total}

O gradiente total (GT) foi utilizado neste trabalho, porque a inclinação magnética possui valores baixos, em torno de $-34^{\circ}$, na latitude $16^{\circ} 15^{\prime} 30^{\prime \prime} \mathrm{S}$ e longitude $42^{\circ} 44^{\prime} \mathrm{W}$ (National Oceanic and Atmospheric Admistration 2019). O resultado do GT é independente da inclinação da magnetização e é particularmente útil em baixas latitudes (Dentith \& Mudge 2014). Foi calculado a partir da raiz quadrada da soma dos quadrados de cada uma das primeiras derivadas direcionais do campo magnético (Figura 5). Este cálculo resulta em valores máximos nas bordas dos corpos, destacando a posição de corpos estreitos, como diques, e as bordas dos corpos maiores (Dentith \& Mudge 2014), sendo útil para realçar limites de fontes magnéticas (Roest, Verhoef \& Pilkington 1992). O resultado do cálculo do GT mostra anomalias magnéticas positivas na porção centronorte da área (Figura 5), aproximadamente coincidentes com o metadiamictito hematítico que tem teor variável de magnetita. Grande anomalia negativa é vista na porção sudeste da área, coincidindo aproximadamente com a localização dos metadiamictitos estéreis (Figura 5).

\subsubsection{Deconvolução de Euler}

Detalhado trabalho de prospecção em superfície e sub-superfície foi executado pela SAM na área estudada, incluindo cerca de 450 furos de sondagem com profundidade suficiente para se atingir a unidade estéril na base do corpo de minério. $\mathrm{O}$ algoritmo de deconvolução Euler 3D (Thompson 1982) foi executado com a finalidade de se comparar os resultados calculados das profundidades das fontes magnéticas com os dados de campo e de furos de sondagem da SAM. Foi utilizado o índice estrutural $\mathrm{N}=1$, correspondente a corpo planar de duas dimensões, que é o tipo de geometria esperado para o depósito. $\mathrm{O}$ processamento gerou 59 soluções, com profundidades entre 0 e $399 \mathrm{~m}$ para as fontes magnéticas, sendo cerca de $60 \%$ das soluções para profundidade de até $200 \mathrm{~m}$, e outros $22 \%$ para profundidade de até $300 \mathrm{~m}$ (Figura 5). As soluções encontradas na Deconvolução de Euler são coerentes com as profundidades do corpo de minério em seção geológica (Figura 3).

\subsubsection{Dados Gamaespectrométricos}

Interpretações baseadas em dados gamaespectrométricos de coberturas sedimentares, rochas intemperizadas ou regolitos, podem ser prejudicadas pela falta de afloramentos de rocha fresca. Na região estudada, solos lateríticos bem desenvolvidos e lateritas geralmente cobrem a maioria dos tipos de rochas e as chapadas são amplamente cobertas por lateritas ricas em minerais de hidróxido de ferro e materiais amorfos contendo ferro (Salgado, Santos \& Paisani 2019). Embora o conteúdo médio dos radioelementos (K, Th, U) medidos para pesquisas por gamaespectrometria seja baixo em formações ferríferas bandadas e nas rochas sedimentares mais comuns (Boyle 1982; Kileen 1979), esses elementos podem mostrar variações significativas nas concentrações em lateritas, solos e rochas intemperizadas. 

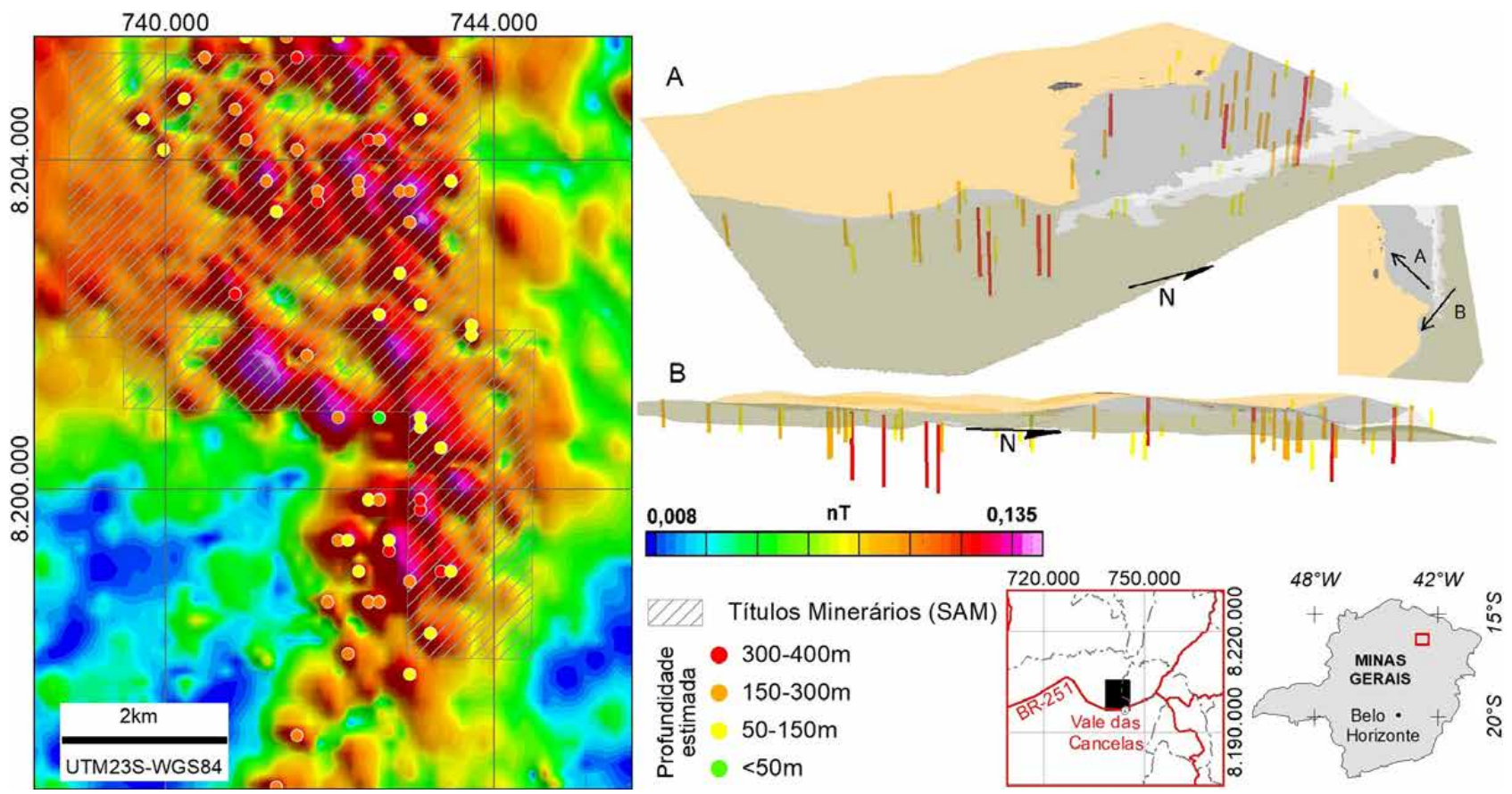

Figura 5 Mapa do Gradiente Total associado às soluções Euler para a área de pesquisa da SAM S.A. A e B. Visualização das profundidades das soluções Euler sobre o mapa geológico da Figura 3; A. visada NW; B. visada EW.

A composição ternária com os canais de potássio, tório e urânio mostra as diferentes proporções destes elementos na superfície (Figura 6A). Neste caso, a imagem mostra que, sobre as coberturas detrito-lateríticas, o potássio já foi praticamente todo lixiviado e só aparece, em tons de magenta, vermelho e amarelo, nas regiões dos vales que circundam estas chapadas. Tons de roxo e magenta mostram a presença de urânio nestas áreas, assim como sobre as coberturas, em tons de ciano. O tório aparece em maiores concentrações nos topos das chapadas, mas também está presente nos vales, nos pontos onde as cores ciano, amarelo e branco aparecem.

Em solos lateríticos e lateritas, o urânio e o tório tendem a ser absorvidos por minerais de argila e hidróxidos de ferro, as lateritas tendem a concentrar altos teores de tório, o urânio pode ser enriquecido nos níveis mais altos do solo e o potássio é o mais móvel dos três radioelementos, tendendo a ser facilmente removido por intemperismo químico em lateritas comuns (Boyle 1982; Wilford, Bierwirth \& Craig 1997). A razão $\mathrm{Th} / \mathrm{K}$ destaca concentrações anômalas de tório relacionadas a coberturas extensas de laterita em chapadas (Figura 6B). As razões U/K distinguem solos colúvio-eluviais nas bordas da chapada (Figura $6 \mathrm{C}$ ), já que o conteúdo de U é superior ao teor de $\mathrm{K}$ nesses solos e pode ser fixado em depósitos ricos em hidróxido de ferro, como nas lateritas e nos solos lateríticos encontrados na região estudada (Wilford, Bierwirth \& Craig 1997).

\subsection{Imagens Landsat 8}

O satélite Landsat 8 carrega o sensor Operational Land Imager OLI, que coleta dados de imagem em 9 bandas espectrais de ondas curtas em uma faixa de $190 \mathrm{~km}$, com uma resolução espacial de $30 \mathrm{~m}$ para todas as bandas, exceto a banda Pan, de $15 \mathrm{~m}$ (United States Geological Survey 2018). Produtos desse sensor têm sido amplamente utilizados para estudos geológicos como mapeamento litológico ou prospecção mineral (Salem \& El Gammal 2015; Zacchi et al. 2010). Neste estudo, a cena 218-71, adquirida durante a estação seca (1/6/2017), foi recortada na região estudada (Figura 7A).

\subsection{1. Índice para Realce de Hematita (IH)}

De acordo com Madeira et al. (1997), trabalhando em índices espectrométricos visíveis baseados no conteúdo de hematita e goethita em solos lateríticos, o índice de vermelhidão geralmente fornece uma boa correlação com o conteúdo de hematita em lateritas. Os autores aplicaram este modelo a uma imagem TM Landsat (TM3) para estimar o conteúdo de hematita nos horizontes do solo, que corresponde a um índice radiométrico chamado por eles de índice para realce de hematita (IH; Madeira et al. 1997). O cálculo do IH, usando as bandas visíveis (bandas 2, 3 e 4) do sensor do satélite Landsat 8 , sugere alta concentração de hematita nas áreas onde há solo exposto (Figura 7B). 

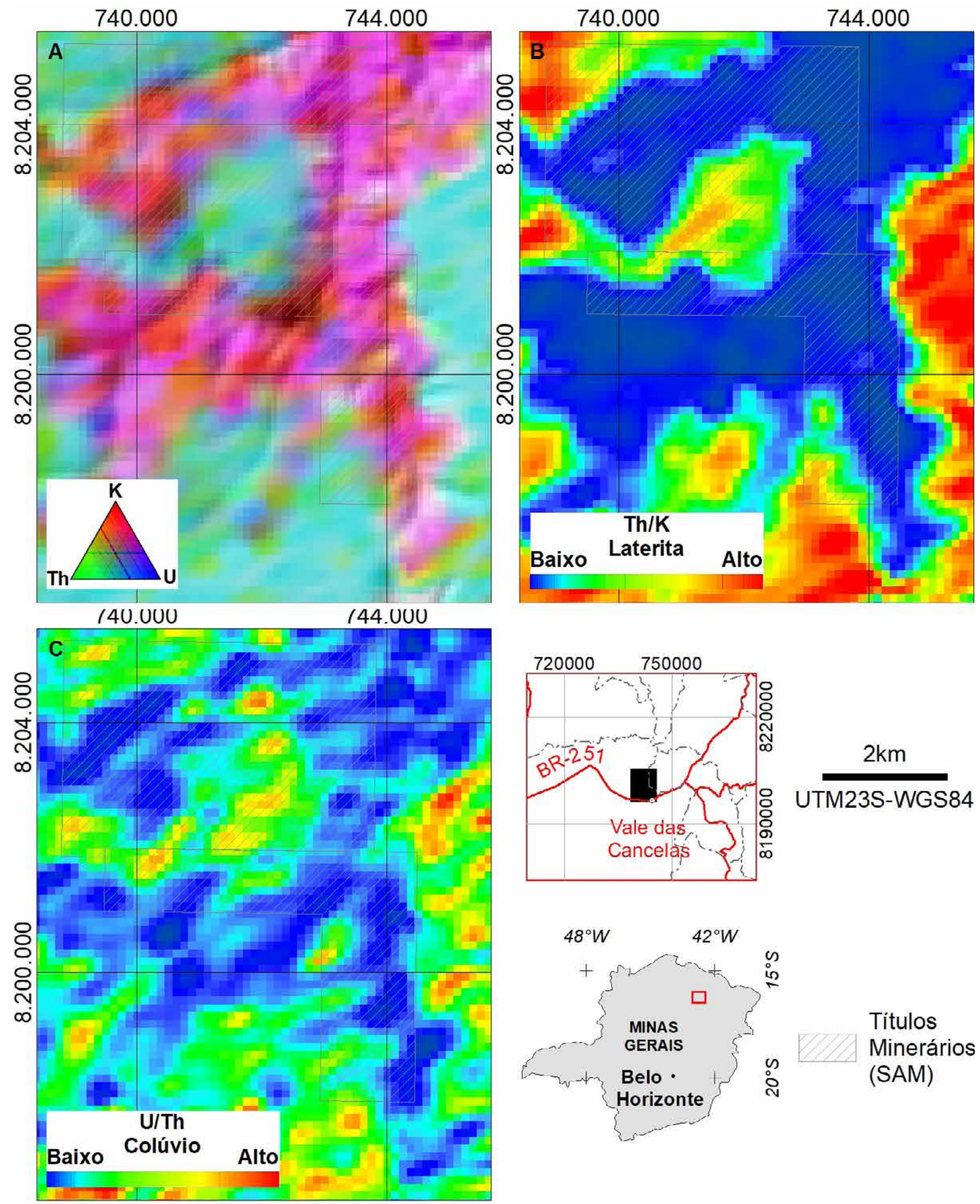

Figura 6 A. Composição ternária com as concentrações de K (vermelho), Th (verde) e U (azul); B. Razão Th/K; C. Razão U/Th. 

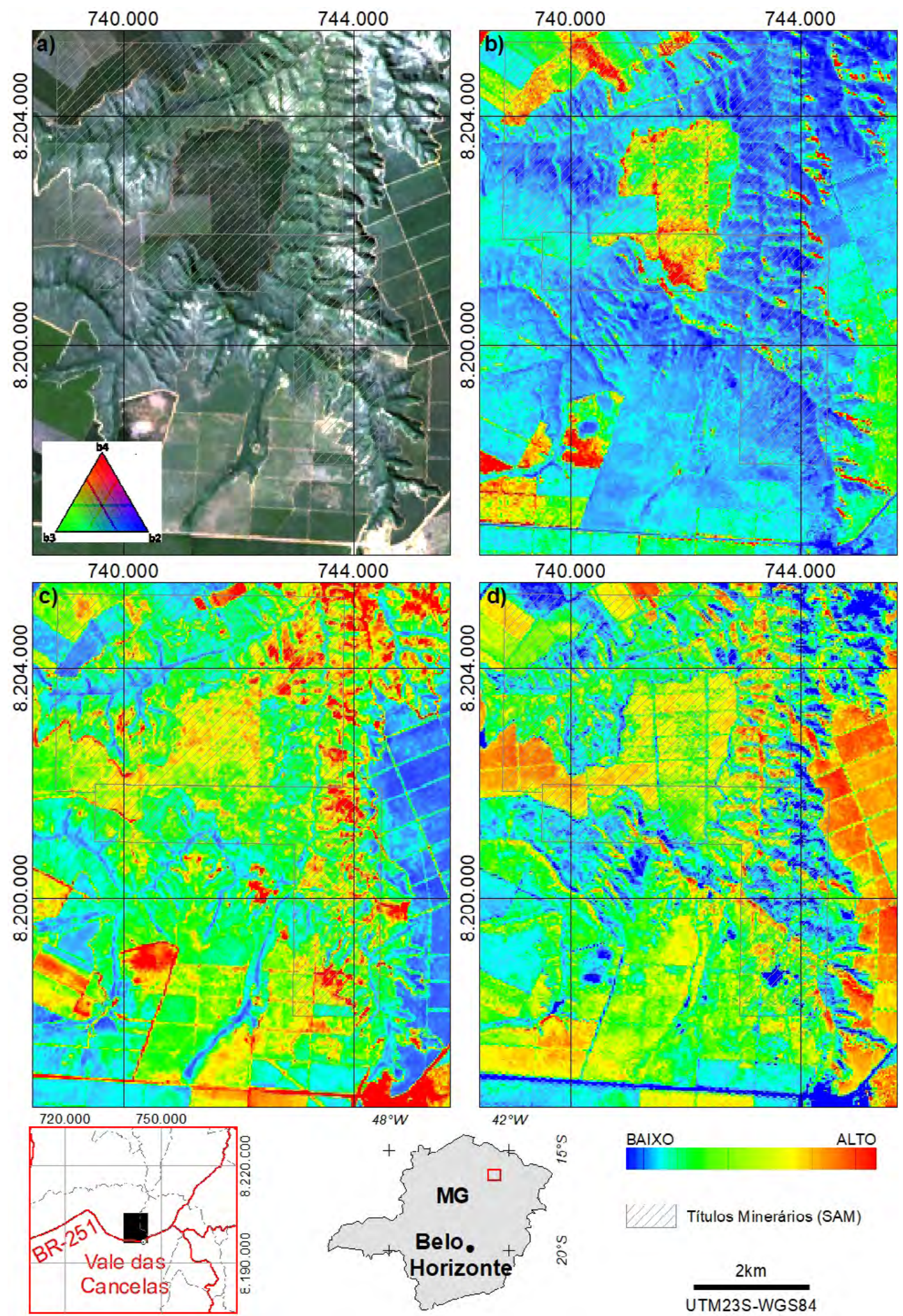

Figura 7 A. Imagem Landsat 8 (RGB 432) mostrando as coberturas que sustentam o relevo circundadas por vales onde afloram as rochas da Formação Nova Aurora; B. Índice para realce de hematita $(\mathrm{IH})$; C. Análise por Componente Principal (ACP) para realce de óxido-hidróxido de ferro; D. APC para argilominerais. 


\subsubsection{Análise por Componente Principal (ACP)}

A Análise por Componente Principal (ACP), resulta da combinação linear dos dados originais e dos vetores próprios aplicada, pixel a pixel, a imagens correlacionadas, fornecendo um novo conjunto de imagens não correlacionadas (Lillesand, Kiefer \& Chipman 2004; Ready \& Wintz 1973). A Análise Seletiva por Componentes Principais (Chavez \& Kwarteng 1989), utilizada neste trabalho, usa apenas um par de imagens selecionadas para mapear contrastes espectrais entre diferentes partes do espectro, com o objetivo de encontrar informações bastante distintas para cada banda, simplificando as imagens, selecionando as informações desejadas e eliminando a redundância. Para isso, é utilizado um par de imagens com correlação moderada $(0,7$ a 0,98$)$ a baixa $(<0,7)$, sendo que o segundo componente gerado traz as informações mais contrastantes (Chavez e Kwarteng 1989). Com o objetivo de destacar óxidos e hidróxidos de ferro, a ACP foi aplicada às bandas 2 e 4 (correlação 0,92; Figura 7C). A primeira componente resultante contém as informações redundantes, como sombreamento topográfico e albedo, enquanto que a segunda componente traz a informação espectralmente contrastante entre elas, nesse caso os compostos de ferro concentrados no metadiamictito. A ACP com as bandas 6 e 7 (correlação 0,96; Figura 7D) foi utilizada para mapear superfícies ricas em minerais com $\mathrm{OH}^{-}$, detectando as elevadas concentrações desses minerais, que indiretamente auxiliam no mapeamento das formacões ferríferas ocultas sob coberturas lateríticas (Zacchi, Silva \& Rolim 2010).

\section{Modelagem Espacial}

\subsection{Caracterização da Formação Ferrífera}

As unidades das formações Serra do Catuni, Nova Aurora e Chapada Acauã Inferior, do Grupo Macaúbas, contêm diamictitos interpretados como depósitos glaciogênicos. A Formação Nova Aurora contém predominantemente metadiamictito, com quartzito e filito subordinados. O portador do minério de ferro da formação é o Membro Riacho Poções, individualizado por Viveiros et al. (1978), que se trata de um metadiamictito hematítico com intercalações de quartzito e filito hematíticos, com espessura estimada em até $600 \mathrm{~m}$ (Grossi-Sad et al. 1997; Viveiros et al. 1978). O contato basal do Membro Riacho Poções é caracterizado por uma zona de cisalhamento dúctil, onde o minério é concentrado por lixiviação, resultando em um itabirito com grande concentração de hematita e magnetita (Figura 4), atingindo quantidade de até $60 \%$ (em volume) da matriz do metadiamictito hematítico. A partir do contato basal, há predominância de metadiamictito hematítico com teor variável de ferro, que decresce rumo ao topo (Vilela et al. 2021).

\subsection{Modelagem dos Dados}

Os dados geofísicos e de sensoriamento remoto foram reclassificados e combinados usando os operadores de lógica fuzzy para modelagem de dados (Bonham-Carter 1994). Os dados significativos de informação escolhidos são: o GT, que mostra anomalias de alta intensidade magnética $\mathrm{e}$, consequentemente, tende a destacar unidades de rocha com conteúdo significativo de minerais de ferro magnético que devem ocorrer na região, como metadiamictitos ricos em hematita magnética e/ou magnetita (Figuras 4 e 8); as razões gamaespectrométricas de $\mathrm{Th} / \mathrm{K}$ (Figura 6B) e U/K (Figura 6C), que realçam as características da superfície relacionadas ao intemperismo, destacando áreas com altas concentrações de Th e U e baixas concentrações de K, relacionadas ao alto teor de hidróxido de ferro em lateritas; o IH (Figura 7B); e os resultados da ACP-Fe, para óxidohidróxido de ferro (Figura 7C) e da ACP-arg, para argilominerais (Figura 7D).

Os dados selecionados foram reclassificados (Figura 8), inicialmente transformando seu intervalo de valores originais em intervalos de 0 (favorabilidade mínima) a 1 (favorabilidade máxima). O operador large foi aplicado às imagens GT, Th/K, U/K, IH e ACP-Fe, enquanto o operador small foi aplicado à ACP-arg e às imagens $\% \mathrm{~K}, \mathrm{Th}_{\mathrm{ppm}}$ e $\mathrm{U}_{\mathrm{ppm}}$. A função large é usada quando os valores mais altos em cada dado correspondem à maior favorabilidade do alvo estudado, enquanto a função small é aplicada se os valores mais baixos em cada dado se referem à maior favorabilidade. Em seguida, as imagens $\mathrm{K}_{\text {small }}$, $\mathrm{Th}_{\text {small }}$ e $\mathrm{U}_{\text {small }} ; \mathrm{Th} / \mathrm{K}_{\text {large }}$ e U/K $/ \mathrm{K}_{\text {large }}$; e $\mathrm{IH}_{\text {large }}$ e ACP-Fe $\mathrm{Fe}_{\text {large }}$ foram combinadas usando o operador soma algébrica fuzzy. Como resultado o operador soma algébrica fuzzy é sempre maior que (ou igual a) o maior valor de membro fuzzy. O efeito é, portanto, "crescente". Neste caso as evidências que favorecem uma hipótese se reforçam e a evidência combinada é mais favorável do que qualquer evidência obtida individualmente (Bonham-Carter 1994).

Finalmente, as imagens resultantes foram combinadas com o operador fuzzy gama (Bonham-Carter 1994; Voll 2020; Figura 8). O índice de importância $\gamma$ (gama), variando entre 0 e 1, define a relevância de cada membro do operador. A modelagem foi feita de forma que maiores valores reflitam maior favorabilidade para a ocorrência de minério de ferro. Depois de uma sequência de testes, foi utilizado o índice de importância $\gamma=0,9$ para a geração do modelo. A imagem resultante mostra locais com maior favorabilidade para a ocorrência de minério de ferro na região de estudo (Figura 9). 


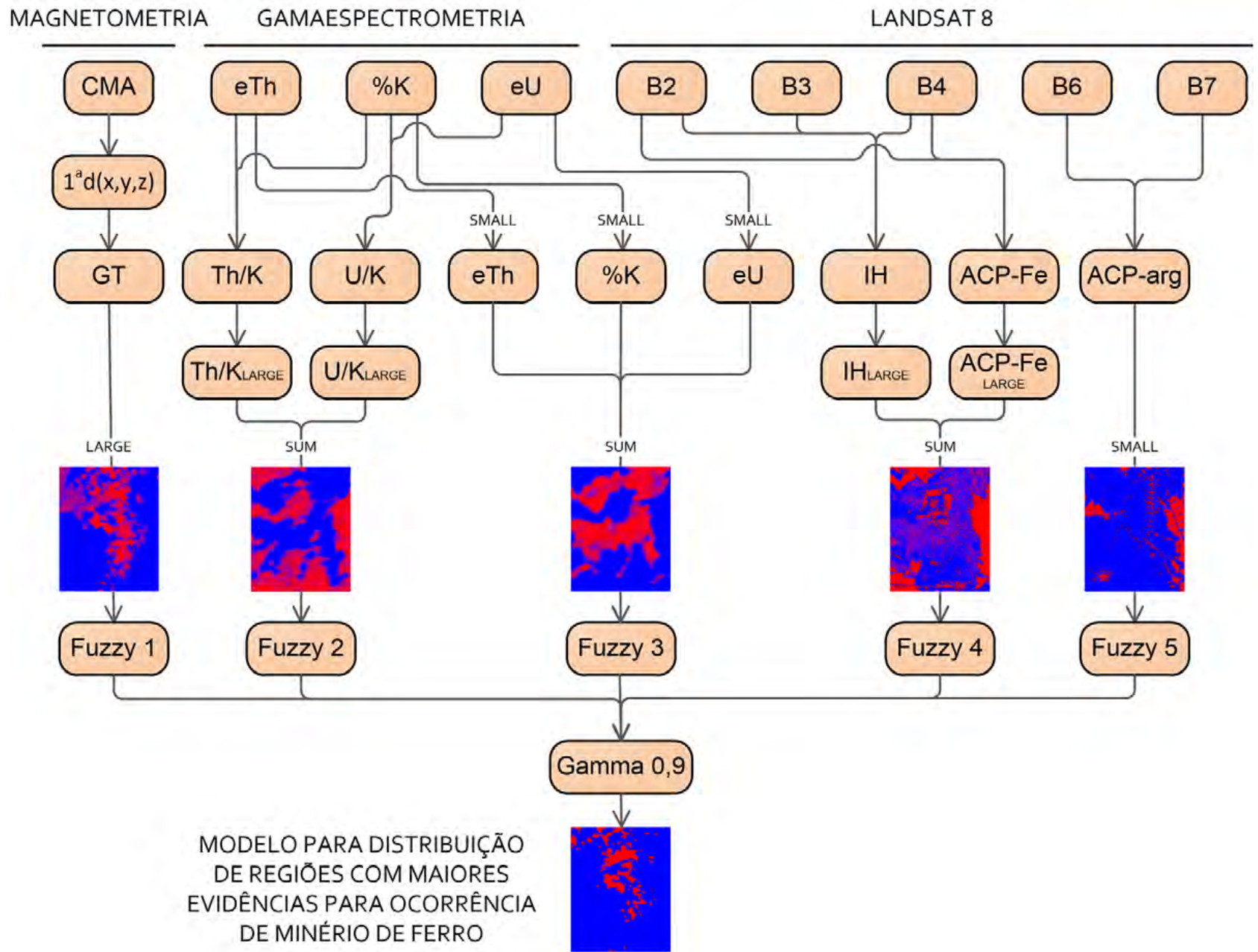

Figura 8 Fluxograma de processo para análise e geração de modelo prospectivo para minério de ferro.

\section{Discussão e Conclusões}

Com o objetivo de rastrear a ocorrência de minério de ferro em uma área de estudo local, largamente oculta por coberturas lateríticas sobre chapadas, foram gerados vários produtos com base em dados de levantamentos aerogeofísicos magnéticos e gamaespectrométricos, além de imagens de satélite Landsat 8 . Como os dados magnéticos revelam locais relativamente profundos com concentrações anômalas de rochas ricas em ferro, também foram empregados dados da gamaespectrometria e sensoriamento remoto para refinar os produtos de integração, melhorando assim a interpretação sobre as chapadas. A vasta extensão de coberturas lateríticas pode mascarar a resposta superficial de rochas ricas em ferro na gamaespectrometria e nas imagens Landsat 8, pois esses sensores coletam apenas a resposta do material da superfície. No entanto, existem afloramentos dispersos de rochas ricas em ferro preservadas da lateritização em chapadas, pois os hidróxidos de ferro são geralmente mais concentrados em lateritas desenvolvidas sobre camadas de metadiamictito ferruginoso e formação ferrífera diamictítica. Portanto, o uso de gamaespectrometria e dados de sensoriamento remoto, integrados aos dados magnéticos, revelou-se eficaz na redução da ambiguidade da informação, porque os dados magnéticos por si só, não são suficientes para delimitar com confiabilidade os locais mais favoráveis para a ocorrência do minério de ferro em superfícies de chapadas.

Como o problema focado (ou seja, pesquisar minério de ferro oculto sob as chapadas amplamente cobertas por laterita) dificulta a aplicação de limites rígidos entre as classes de dados, a lógica fuzzy foi aplicada a fim de produzir modelos orientados pelo conhecimento (An, Moon, W.M. \& Rencz 1991; Bonham-Carter 1994; Porwal et al. 2015). O modelo utilizado para este estudo de caso foi aquele aplicado por Voll, Silva \& Pedrosa-Soares (2020) em escala regional, com o objetico de rastrear rochas ricas em ferro. 

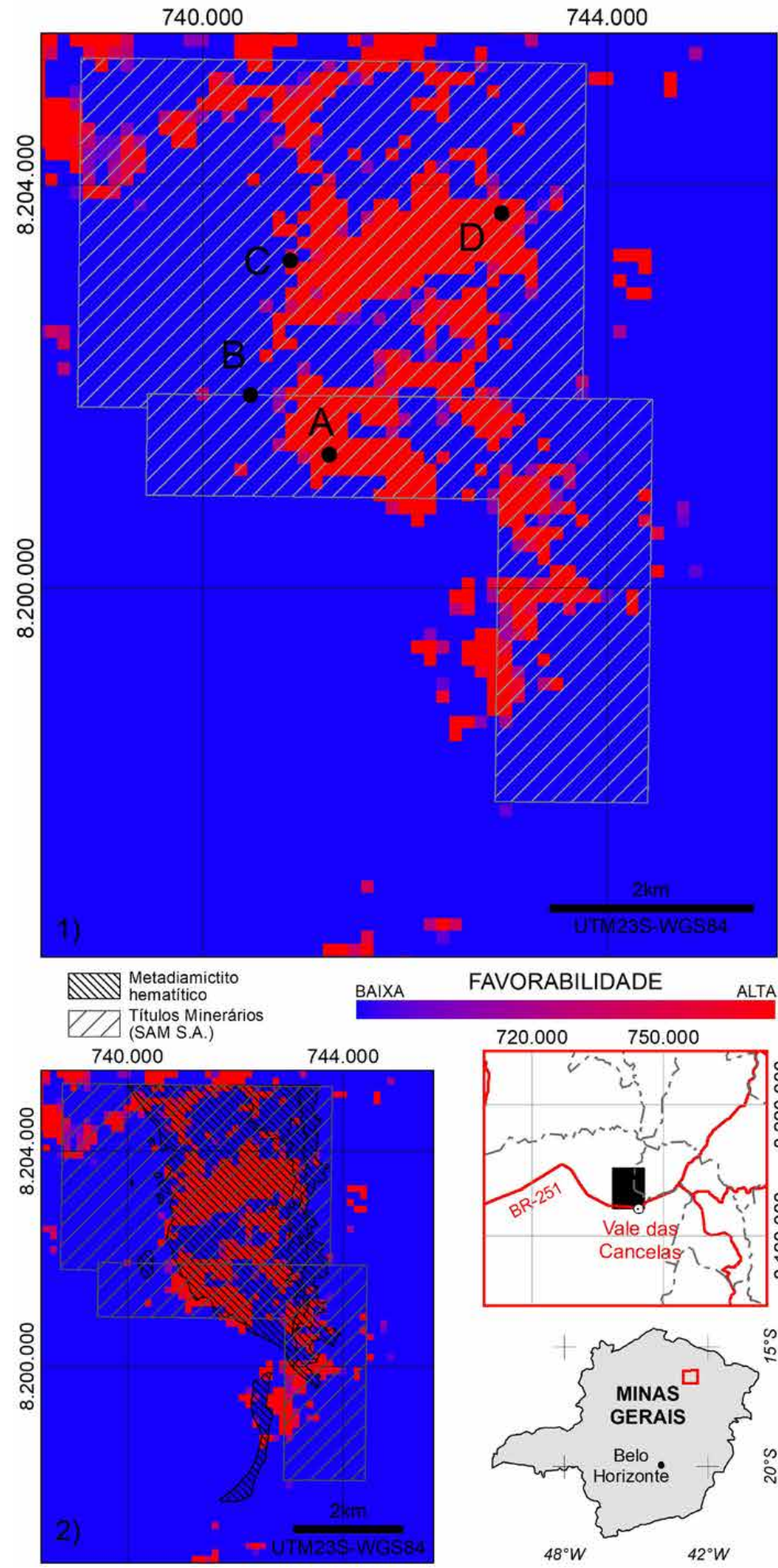

FAVORABILIDADE

$720.000 \quad 750.000$
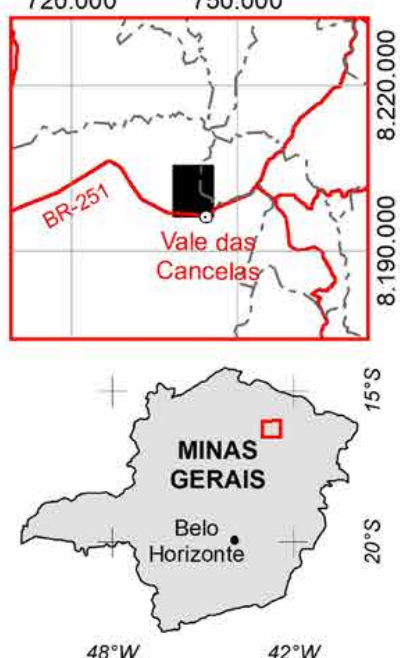
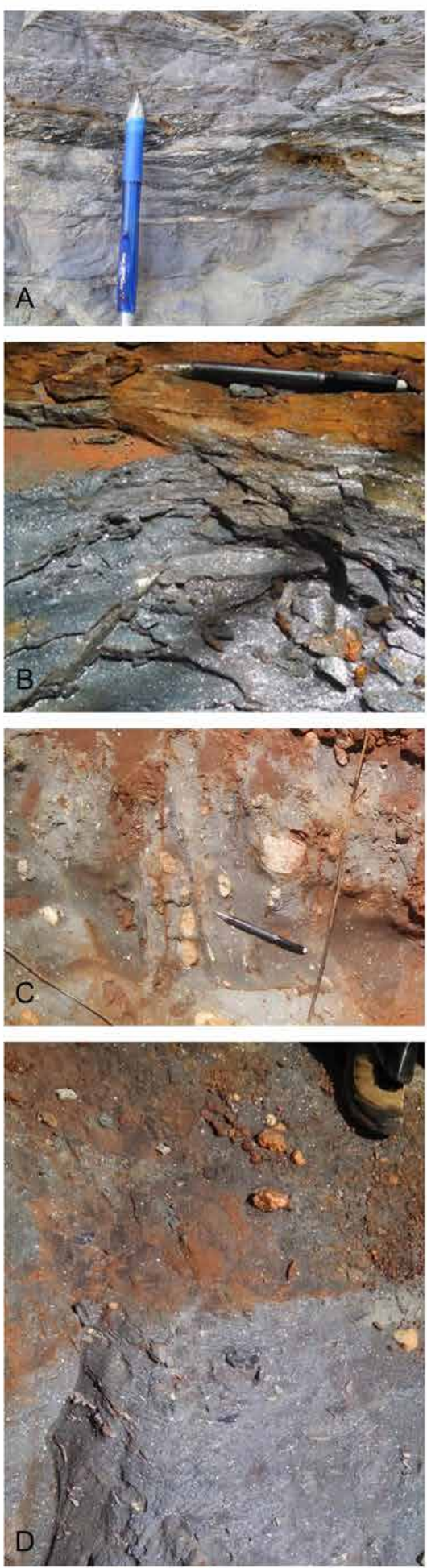

Figura 9 Mapa 1: Modelo prospectivo para minério de ferro mostrando áreas com alta favorabilidade à prospecção de minério de ferro em vermelho e localização dos pontos A, B, C e D (afloramentos de metadiamictito hematítico descritos em campo); Mapa 2: Modelo prospectivo para minério de ferro mostrando as áreas de requerimento de lavra da SAM S.A. (Agência Nacional de Mineração 2019) e os limites da unidade que contém o minério (modificado de Sul Americana de Metais 2013); A-D. Afloramentos de metadiamictito hematítico (UTM23S-WGS84 A - 741.246E, 8.201.332N; B - 740.468E, 8.201.919N; C - 740.858E, 8.203.248N; D - 742.962E, 8.203.719N). 
Esse modelo mostrou-se eficiente para estudos locais. $\mathrm{O}$ resultado é um modelo integrado que mostra os locais mais favoráveis para a ocorrência de minério de ferro na região estudada (Figura 9, Mapa 1). O modelo final proposto mostra que as maiores e mais bem definidas áreas ricas em ferro estão localizadas nas porções noroeste e centro da região estudada, onde predominam os tabuleiros com coberturas lateríticas, escondendo todos os tipos de rochas subjacentes.

O modelo apresentado mostra correspondências consistentes entre os principais locais favoráveis e os dados utilizados para validação, como informações de campo, descrições compiladas da literatura e afloramentos localizados em mapas geológicos, bem como os registros oficiais de licenças para prospecção de ferro da SAM nesta área (Agência Nacional de Mineração 2019).

O modelo se mostrou coerente com mapa geológico cedido pela empresa, que não mostra as coberturas lateríticas (Figura 3), assim como também se mostraram coerentes as profundidades estimadas para as fontes magnéticas geradas pela deconvolução de Euler (Figura 7), quando comparadas com as espessuras do corpo de minério mostradas nas seções geológicas (baseadas em furos de sondagem) apresentadas na Figura 3. Os pontos de controle coletados em campo mostram correspondência entre o modelo e a ocorrência do minério (Figura 9).

A integração de dados efetuada mostra que, mesmo considerando as grandes chapadas cobertas por lateritas ferruginosas, é possível identificar e delimitar locais favoráveis à concentração de minério de ferro sob esses platôs. O modelo apresentado por Voll, Silva \& Pedrosa-Soares (2020) se mostrou aplicável em escala local e os procedimentos apresentados para integração de dados e modelos resultantes se mostraram úteis para ajudar na criação de modelos de prospecção mais detalhados aplicados ao Membro Riacho Poções e suas extensões ocultas por coberturas lateríticas sobre chapadas. Esse modelo mostrou ser eficientemente aplicado a escalas diversas (Voll, Silva \& Pedrosa-Soares 2020), podendo ser utilizado em várias situações em outras regiões, não apenas na área de estudo. Por ser uma modelagem orientada pelo conhecimento, pode ser adaptada para vários propósitos, de acordo com objetivos distintos de pesquisa e necessidades de trabalho. É um modelo que evolui a partir da compreensão do fenômeno a ser estudado, o que o torna versátil para muitas outras abordagens.

\section{Agradecimentos}

Ao $\mathrm{CNPq}$ e à CODEMIG-CODEMGE pelo apoio financeiro. À Companhia Sul Americana de Metais (SAM) pela cessão de informações geológicas para a elaboração deste estudo de caso. Eliane Voll agradece à CAPES pela bolsa de doutorado, e os co-autores agradecem ao CNPq por suas bolsas de produtividade em pesquisa. Os autores agradecem ao revisor por seus comentários, correções e sugestões que muito nos ajudaram a melhorar o manuscrito anterior deste artigo, e ao Dr. Gustavo Luiz Campos Pires, por seu trabalho editorial.

\section{Referências}

Agência Nacional de Mineração 2019, Sistema de Informações Geográficas da Mineração-SIGMINE. viewed 12 April 2019, $<$ http://www.anm.gov.br/assuntos/ao-minerador/sigmine>.

An, P., Moon, W.M. \& Rencz, A.N. 1991, 'Application of fuzzy set theory for integration of geological, geophysical and remote sensing data', Canadian Journal of Exploration Geophysics, vol. 27 , no. 1, pp. 1-11.

Andrade, L.B., Silva, A.M. \& Souza Filho, C.R. 2014, 'Nickel prospective modelling using fuzzy logic on Nova Brasilândia Metasedimentary Belt, Rondônia, Brazil' Revista Brasileira de Geofísica, vol. 32, no. 3, pp. 419-31. https://doi.org/10.22564/ rbgf.v32i3.533.

Bonham-Carter, G.F. 1994, Geographic Information Systems for Geoscientists: Modelling with GIS. Pergamon Press, Ontario. ISBN: 0080418678 .

Boyle, R.W. (ed.) 1982, Geochemical prospecting for thorium and uranium deposits. Developments in Economic Geology, vol. 16, Elsevier Scientific Publishing Company, Amsterdam. ISBN: 978-0-444-42070-1

Chavez Jr., P.S. \& Kwarteng, A.Y. 1989, 'Extracting spectral contrast in Landsat Thematic Mapper image data using selective principal component analysis', Photogrammetric Engineering and Remote Sensing, vol. 55, no. 3, pp. 339-48.

Companhia de Desenvolvimento de Minas Gerais - Secretaria de Desenvolvimento Econômico - Companhia de Pesquisa de Recursos Minerais 2006, Levantamento aerogeofísico de Minas Gerais, Programa 2005/2006. Área 8: Minas Novas - Riacho dos Machados - Espinosa. Relatório final do levantamento e processamento de dados magnetométricos e gamaespectrométricos. vol. 1 - Texto técnico. Belo Horizonte.

Crósta, A.P., Bicho, C.P. \& Ferreira, J.F.F. 1994, 'Image processing applied to aerogeophysics: an important tool for geological mapping and exploration in the Brazilian Amazon',.paper presented to the 10th Thematic Conference on Geologic Remote Sensing, San Antonio, Texas, vol. 2, pp. 539-50.

Dentith, M. \& Mudge, S.T. 2014, 'Electrical and electromagnetic methods' in M. Dentith \& S.T. Mudge (eds), Geophysics for the Mineral Exploration Geoscientist, Cambridge University Press, pp. 235-350. https://doi.org/10.1017/ cbo9781139024358.007. ISBN: 9780521809511

Grossi-Sad, J.H., Lobato, L.M., Pedrosa-Soares, A.C. \& SoaresFilho, B.S. (eds) 1997, Projeto Espinhaço em CD-ROM, CODEMIG, Belo Horizonte.

Kileen, PG. 1979, 'Gamma-ray spectrometric methods in uranium exploration - application and interpretation. Geophysics and Geochemistry in the Search for Metallic Ores', Geological Survey of Canada, Economic Geologic Report, vol. 31, pp. 163-229. https://doi.org/10.4095/106037 
Lillesand ,T.M., Kiefer, R.W. \& Chipman, J.W. 2004, Remote Sensing and Image Processing. 5th edn. John Wiley and Sons. ISBN: 0-471-15227-7

Madeira, J.S., Bedidi, A., Cervelle, B., Pouget, M. \& Flay, N. 1997, 'Visible spectrometric indices of hematite (Hm) and goethite $(\mathrm{Gt})$ content in lateritic soils: The application of a Thematic Mapper (TM) image for soil-mapping in Brasilia', International Journal of Remote Sensing. vol. 18, no. 13, pp. 2835-2852. https://doi.org/10.1080/014311697217369

Mourão, M.A.A. \& Grossi Sad, J.H. 1997, 'Geologia da Folha Padre Carvalho, Minas Gerais', in J.H. Grossi-Sad L.M. Lobato, A.C. Pedrosa-Soares \& B.S. Soares-Filho (eds), Projeto Espinhaço em CD-ROM, CODEMIG, Belo Horizonte, pp. 315-418.

National Oceanic and Atmospheric Admistration 2019, Magnetic Field Calculators, viewed 15 July 2019, <https://www.ngdc. noaa.gov/geomag/>.

Pedrosa-Soares, A.C., Babinski, M., Noce, C.M., Martins, M., Queiroga, G. \& Vilela, F.T. 2011, 'The Neoproterozoic Macaúbas Group (Araçuaí orogen, SE Brazil) with emphasis on the diamictite formations' in Arnaud, E., Halverson, G.P. \& Shields-Zhou, G. (eds). The Geological Record of Neoproterozoic Glaciations. Geological Society of London, Memoir, vol. 36, no. 49, pp. 523-34. https://doi.org/10.1144/ M36.49.

Porwal, A., Deb Das, R., Chaudhary, B., Gonzalez-Alvarez, I. \& Kreuzer, O. 2015, 'Fuzzy inference systems for prospectivity modeling of mineral systems and a case-study for prospectivity mapping of surficial Uranium in Yeelirrie Area, Western Australia', Ore Geol. Rev., vol. 71, pp. 839-52. https://doi. org/10.1016/j.oregeorev.2014.10.016

Raines, G.L., Sawatzky, D.L. \& Bonham-Carter, G.F. 2010, 'Incorporating Expert Knowledge - New fuzzy logic tools in ArcGIS 10', ArcUser Spring 2010, pp. 8-13. viewed 5 October 2019, <https:/www.esri.com/news/arcuser/0410/ files/fuzzylogic.pdf $>$.

Ready, P.J. \& Wintz, P.A. 1973, 'Information extraction, SNR improvement and data compression in multispectral imagery', IEEE Transactions on Communications, COM-21, no. 10, pp. 1123-31.

Roest, W. R., Verhoef, J. \& Pilkington, M. 1992, 'Magnetic interpretation using the 3D analytic signal', Geophysics, vol. 57, no. 1, pp. 116-25. https://doi.org/10.1190/1.1443174

Saadi, A. 1995, 'A geomorfologia da Serra do Espinhaço em Minas Gerais e de suas margens', Geonomos, vol. 3, no. 1, pp. 41-63. https://doi.org/10.18285/geonomos.v3i1.215.

Salem, S.M. \& El Gammal, E.A. 2015, 'Iron ore prospection East Aswan, Egypt, using remote sensing techniques', The Egyptian Journal of Remote Sensing and Space Sciences, vol. 18, pp. 195-206. https://doi.org/10.1016/j.ejrs.2015.04.003

Salgado, A.A.R., Santos, L.J.C. \& Paisani, J.C. 2019, The Physical Geography of Brazil: Environment, Vegetation and Landscape, Springer Nature. ISBN 978-3-030-04333-9
Sul Americana de Metais S.A. 2013. Mapa Geológico-Estrutural Integrado com Sondagem e Topografia - Bloco 8, Alvarás de Pesquisa 831.028/2007-831.029/2007, Relatório Técnico, SAM - Sul Americana de Metais S.A.

Thompson, D.T. 1982, 'EULDPH: A new technique for making computer-assisted depth estimates from magnectic data', Geophysics, vol. 47, pp. 31-37.

United States Geological Survey 2018, Landsat 8 Data Users Handbook, viewed 3 April $2019<$ https://www.usgs.gov/ media/files/landsat-8-data-users-handbook $>$.

Vilela, F.T. 2010, 'Caracterização de metadiamictitos ferruginosos da Formação Nova Aurora (Grupo Macaúbas, Orógeno Araçuaí) a Oeste de Salinas, MG', Masters Thesis, Universidade Federal de Minas Gerais, Belo Horizonte.

Vilela, F.T., Pedrosa-Soares, A.C., Carvalho, M.T.N., Arimatéia, R., Santos, E. \& Voll, E. 2014, 'Metalogênese da Faixa Araçuaí: o Distrito Ferrífero Nova Aurora (Grupo Macaúbas, norte de Minas Gerais) no contexto dos recursos minerais do Orógeno Araçuaí' in M.G. Silva, M.B. Rocha Neto, H. Jost \& R.M. Kuyumjian (eds), Metalogênese das Províncias Tectônicas Brasileiras, CPRM, pp. 415-30.

Vilela, F.T., Pedrosa-Soares, A., Babinski, M., Lana, C., Trindade, R.I.F. \& Santos, E. 2021. 'Diamicititic iron formation (DIF) deposits of the Neoproterozoic Nova Aurora District (Macaúbas Group, Southeast Brazil)', Journal of South American Earth Sciences, 112:103614. https://doi. org/10.1016/j.jsames.2021.103614

Viveiros, J.F.M., Sá, E.L., Vilela, O.V., Santos, O.M., Moreira, J.M.P., Holder-Neto, F. \& Vieira, V.S. 1978, 'Geologia dos vales dos rios Peixe Bravo e Alto Vacaria, norte de Minas Gerais', 30th Congresso Brasileiro de Geologia, Recife, vol. 1, no. 243, pp. 243-54.

Voll, E., Silva, A.M. \& Pedrosa-Soares, A.C. 2020, 'Tracking iron-rich rocks beneath Cenozoic tablelands: An integration of geological, airborne geophysical and remote sensing data from northern Minas Gerais State, SE Brazil', Journal of South American Earth Sciences, 101:102604. https://doi. org/10.1016/j.jsames.2020.102604

Wilford, J.R., Bierwirth, P.N. \& Craig, M.A. 1997, 'Application of airborne gamma-ray spectrometry in soil/regolith mapping and applied geomorphology', AGSO Journal of Australian Geology \& Geophysics, vol. 17, no. 2, pp. 201-16.

Zacchi, E.N.P., Silva, A.M. \& Rolim, V.K. 2010, 'Análise integrada de dados multifonte e sua aplicação no mapeamento geológico das formações ferríferas da Serra de Itapanhoacanga, Alvorada de Minas, MG', Revista Brasileira de Geofísica, vol. 28, no. 4, pp. 643-56. https://doi.org/10.1590/S0102261X2010000400009

\section{Como citar:}

Voll, E., Silva, A.M., Pedrosa-Soares, A., Arimatéia, R. \& Vilela, F.T. 2021, 'Modelagem de Dados Geofísico-espaciais por Lógica Fuzzy Aplicada ao Distrito Ferrífero Nova Aurora, Norte de Minas Gerais', Anuário do Instituto de Geociências, vol. 44: 36153. https://doi. org/10.11137/1982-3908_2021_44_36153 